\title{
Sepsis from Neonatal Mastitis and Breast Abscess
}

\author{
Seung Eun Lee, Jeongho Lee, Eun Sook Suh \\ Department of Pediatrics, Soonchunhyang University Hospital, Seoul, Korea
}

\begin{abstract}
Although some full-term neonates suffer from mastitis or a breast abscess, mastitis is not common in neonates and concurrent bacteremia is a rarely seen. We report the case of a 30-day old boy of African ethnicity, who was a full-term infant and appropriate for gestational age. At birth, he had bilateral palpable breast masses. He had high fever as well as swelling and nipple discharge in both breasts 30 days after birth. An ultrasonography of the breast showed masses with septa in both breasts. The breast masses were red in color, swollen, and felt hot to the touch. We incised the mass and performed a culture of the discharge. Staphylococcus epidermidis was detected in both blood and the breast discharge. In conclusion, the infant experienced neonatal bacteremia that originated from a breast abscess.
\end{abstract}

Keywords: Mastitis of newborn; Breast abscess

\section{INTRODUCTION}

Mastitis during the neonatal period is an uncommon infection. Typically, mastitis can be seen in infants 2 months or older [1]. It is more common in girls, and longer duration of physiological breast hypertrophy is associated with females $[1,2]$. The main organism causing neonatal mastitis is Staphylococcus aureus, but in rare cases, gram-negative bacteria such as Escherichia coli, Pseudomonas aeruginosa, Proteus mirabilis, and Salmonella species can cause neonatal mastitis [1-5]. Some studies found that Group A and B Streptococcus, and S. epidermidis can cause neonatal mastitis and breast abscesses. In term neonates, breast hypertrophy or nipple discharge is a physiologic change that is induced by maternal hormones. However some pathogenic bacteria on the skin and/or mucous membrane can spread to the breast parenchyma through the nipple. Moreover, hematogenous spread of gram-negative bacteria is possible.

Infants with mastitis usually present with symptoms of local infection such as swelling, erythema, tenderness and induration. Some infants show systemic symptoms including fever, irritability, lethargy and vomiting [1]. In our study, we review the case of a 30-day old infant with mastitis. We describe his presentation, the extent of our evaluations, and treatment.

\section{CASE REPORT}

In our pediatric clinic, a 30-day old boy presented with a fever of $>38^{\circ} \mathrm{C}$ for 1 day ago. He did not have a family history of immunocompromised condition or infection. The infant was born by Cesarean section at a gestational age of 40 weeks and 6 days. He had undergone all routine vaccinations, including those for tuberculosis and hepatitis B.

At birth, the patient had bilateral swelling of the breast, but no signs of local infection such as redness, hotness, tenderness, or nipple discharge were seen. However, over time, the swelling of the breasts worsened, and the parents suspected that the boy had a breast mass (Fig. 1). In course of history taking, we found that the parents of baby didn't squeeze the breast mass and apply the ointment on the breast.

When he presented to our hospital, the infant's breasts felt warm, and the skin of the breast was red. He had high fever of $>38^{\circ} \mathrm{C}$, but no other symptoms except mastitis. He was admitted to our hospi- 


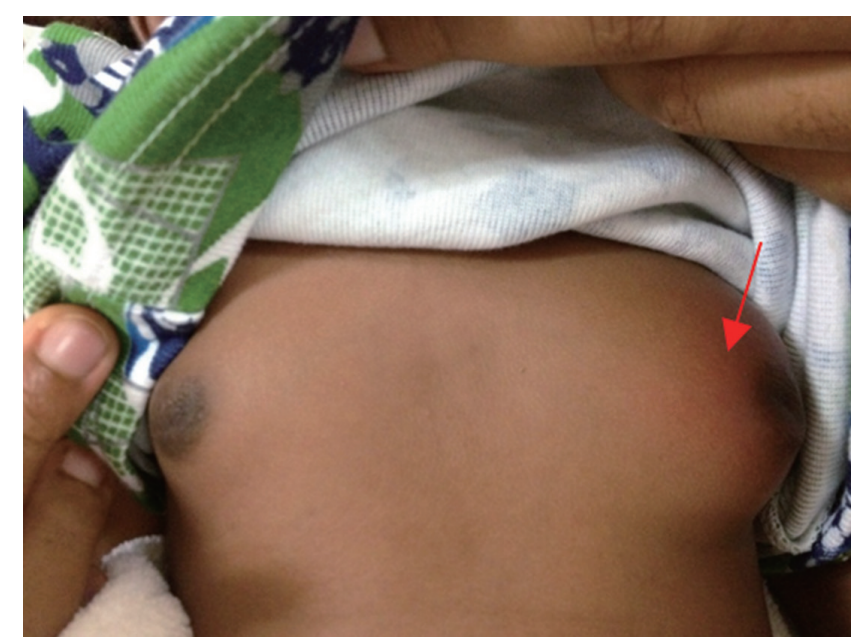

Fig. 1. The patient had breast masses that were red and felt hot to the touch on both sides (arrow).

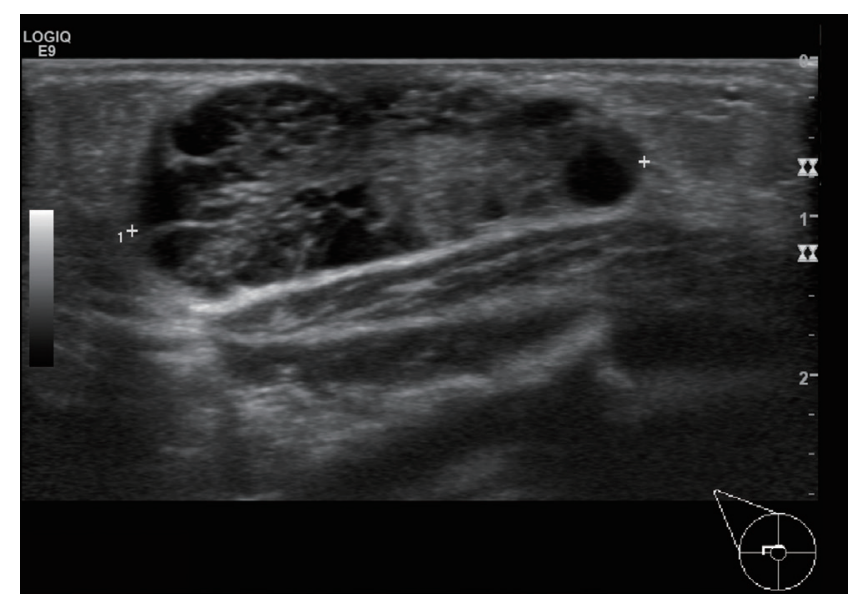

Fig. 2. Ultraconography showed a loculated complex echoic mass with a size of $3.3 \mathrm{~cm}$, in the infant's right breast.

tal and underwent an ultrasonography of the breast and blood tests, including a blood culture to evaluate systemic bacteremia.

A complete blood count with differential was performed. The patient's white blood cell was not elevated $\left(9,000\right.$ cells $\left./ \mathrm{mm}^{3}\right)$, whereas erythrocyte sedimentation rate $(16 \mathrm{~mm} / \mathrm{hr})$ and C-reactive protein $(1.17 \mathrm{mg} / \mathrm{dL})$ level were mildly elevated. And the absolute neutrophil count $(4,167 / \mu \mathrm{L})$ was not decreased, so there was no evidence of patient's immune deficiency. We found bilateral masses in the subareolar areas with size of $3.3 \mathrm{~cm}$ (right) and $4.2 \mathrm{~cm}$ (left) (Figs. 2, 3). The masses contained septa and complex echogenic material. On the left side, the skin was thickening, and subcutaneous fat infiltration near the mass was seen, indicating inflammation of the breast.

Initially, an intravenous antibiotic (cefotaxime; $50 \mathrm{mg} / \mathrm{kg}$ every

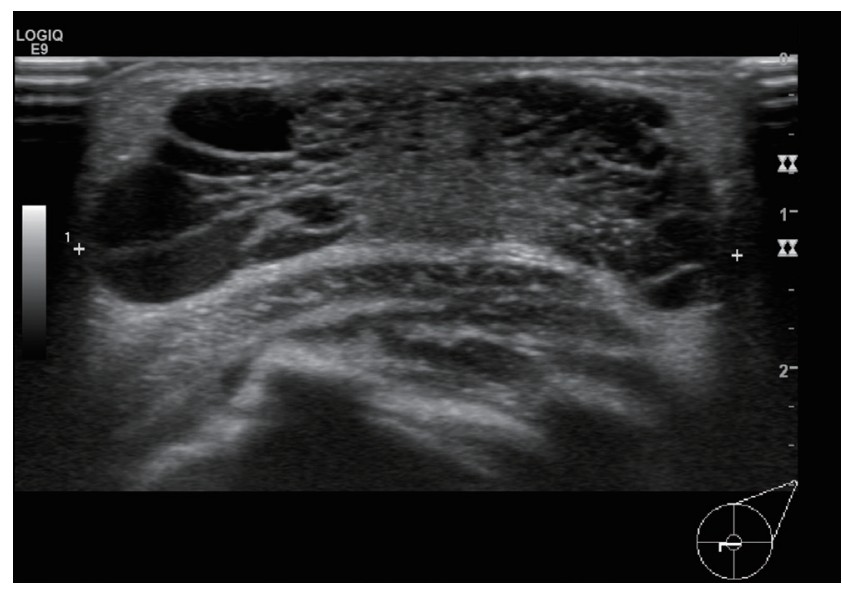

Fig. 3. Ultrasonography showed an abscess with a size of $4.2 \mathrm{~cm}$ in the left breast.

8 hours) was administered. However, the infant continued to have a fever, and the masses was getting larger. On the 4th day of his hospitalization, we incised and drained the mass (Fig. 4A, B).

A blood culture was performed and revealed colonization with methicillin-resistant S. epidermidis (MRSE). Hence, therapy with vancomycin (10 mg/kg every 6 hours) for MRSE bacteremia was initiated. Two days after the drainage, the infant's fever subsided, and the breast mass was getting smaller (Fig. 5A, B). We detected S. epidermidis in the breast discharge. Four days later, a second blood culture test showed no bacterial growth. Hence, the patient was discharged.

\section{DISCUSSION}

Mastitis is an infection of the breast tissue. In neonates, breast enlargement results from maternal hormones acquired through the placenta. It occurs with the same frequency in boys and girls during the first 2 weeks after birth. Thereafter, it is more common in girls, and the girls:boys ratio is 2:1 [1,2].

It is known that mastitis in neonates commonly originates from an infection through the skin. Typically, neonatal mastitis only causes local infection, which results in symptoms such as tenderness, warmth and nipple discharge. In rare cases, mastitis causes systemic symptoms and bacteremia.

In our case, the infant already had breast masses at birth. However, he neither had signs of local infection nor systemic symptoms. Over time, the mass became enlarged, and systemic symptoms emerged. Treatment with third-generation cephalosporins was unsuccessful because the antibiotics were changed and the mass was 

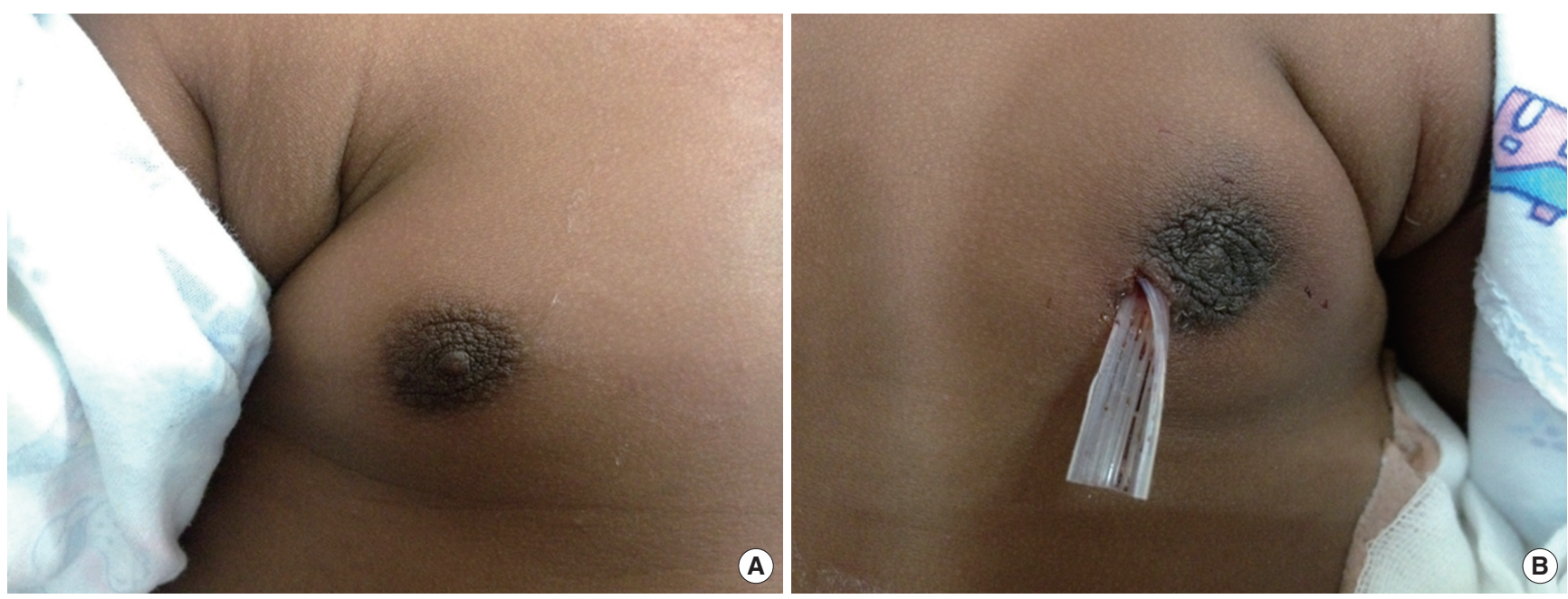

Fig. 4. Four days after initiating therapy with intravenous antibiotics, the masses were incised and drained. (A) Right side of breast. (B) Left side of breast.
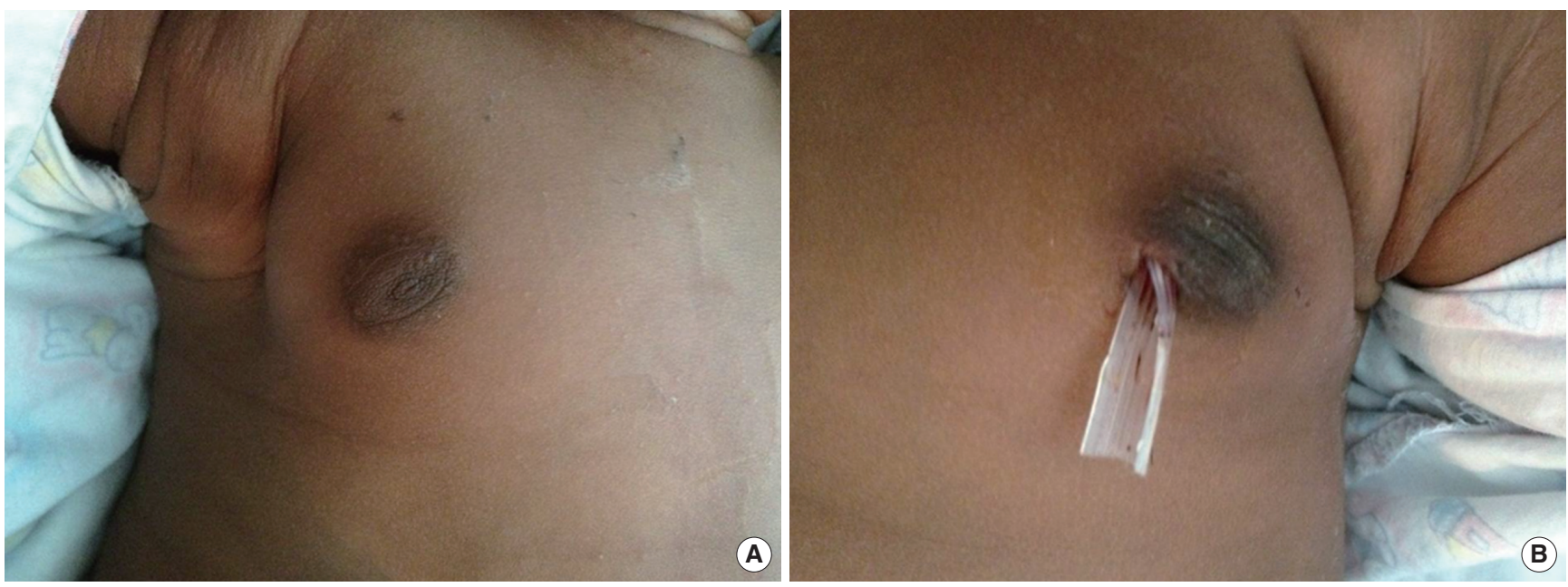

Fig. 5. Two days after the drainage, the breast masses were getting smaller. (A) Right side of breast. (B) Left side of breast.

surgically drained.

Al Ruwaili and Scolnik [1] studied 33 neonates with mastitis and suggested that evaluations such as gram stain, culture and ultrasound were not necessary. It is important to assess the patient's history and to perform a physical examination to distinguish mastitis from physiological hypertrophy. In the case of physiological breast hypertrophy, no tenderness or discharge is typically seen. If present, the color of the discharge is milky rather than purulent. If any continuous signs of local infection (lasting for a few days) are observed, or if the patients have systemic symptoms, they should be further evaluated, and tests including blood culture and, ultrasound should be performed. Because neonatal mastitis can cause bacterial sepsis, and since some cases of mastitis result in abscess formation, surgical drainage is required.
Hence, we should consider evaluating neonates who present with breast swelling using a surgical approach. Moreover, treatment with systemic antibiotics might be required.

\section{REFERENCES}

1. Al Ruwaili N, Scolnik D. Neonatal mastitis: controversies in management. J Clin Neonatol 2012;1:207-10.

2. Walsh M, McIntosh K. Neonatal mastitis. Clin Pediatr (Phila) 1986;25: 395-9.

3. Faden H. Mastitis in children from birth to 17 years. Pediatr Infect Dis J 2005;24:1113.

4. Stricker T, Navratil F, Sennhauser FH. Mastitis in early infancy. Acta Paediatr 2005;94:166-9.

5. Stauffer WM, Kamat D. Neonatal mastitis. Pediatr Emerg Care 2003;19: 165-6. 